IAU Colloquium 190 on Magnetic Cataclysmic Variables

ASP Conference Series, Vol. 315, 2004

Sonja Vrielmann 8 Mark Cropper, eds.

\title{
Thermal-timescale mass transfer and magnetic CVs
}

\author{
Klaus Schenker, Graham A. Wynn \& Roland Speith \\ Theoretical Astrophysics Group, Department of Physics and Astronomy, \\ University of Leicester, University Road, Leicester LE1 7RH, U.K.
}

\begin{abstract}
We investigate the spin evolution of the unusual magnetic CV AE Aqr. As a prototype for a potentially large population of CVs subject to a thermally unstable phase of mass transfer, understanding its future is crucial. We present a new definition of the magnetospheric radius in terms of the white dwarf's spin period, and use this along with numerical simulations to follow the spin evolution of $\mathrm{AE}$ Aqr. We also present preliminary SPH results suggesting the existence of a stable propeller state. These results highlight the complexity of MCVs and may provide an improved understanding of the evolution of all types of CVs.
\end{abstract}

\section{Introduction}

The evolutionary relations among subclasses of magnetic and non-magnetic cataclysmic variables (MCVs and CVs respectively) have been studied for quite some time. Nevertheless, our understanding of these relations remains incomplete, caused largely by serious selection effects which prevent completeness of the samples. Here we consider the future evolution of the peculiar MCV AE Aqr. Its future spin evolution and equilibrium states could clear much of the current confusion, as this binary has been identified as member of a potentially large group of post-thermal timescale mass transfer systems (Schenker et al. 2002). One consequence of this is the prediction of a relatively large population of MCVs at long orbital periods. Such a population has not been detected. We consider the possibility that this could be because these long period MCVs occupy spin equilibria which significantly hamper accretion onto the white dwarf (WD), causing them to have been overlooked or misclassified.

\subsection{Thermal-timescale mass transfer}

We first consider the origin of CVs (Figure 1). The progenitor of the WD starts off as the more massive star in a relatively wide binary, which has evolved through various stages of (single) stellar evolution forming a degenerate core in its centre. During the AGB phase the star swells and fills its Roche lobe. A subsequent phase of common envelope evolution leaves the system with the exposed core (pre-WD) and the largely unaffected secondary in a close orbit. In order to be a future CV this system has to become semi-detached. This can be achieved by angular momentum loss (AML) shrinking the orbit or nuclear evolution increasing the secondary's radius. The latter channel can lead to a 


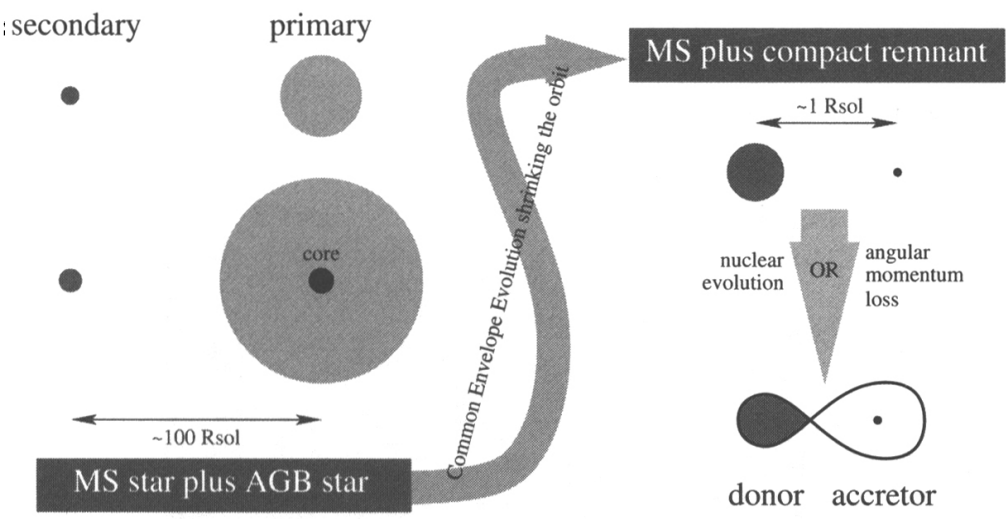

Figure 1. Schematic for the formation of CVs. Note that even systems brought into contact by nuclear evolution in the pre-CV phase (right column) can later turn into angular momentum loss driven semidetached binaries with a red dwarf donor. If (in contrast to the sketch) the donor is initially more massive than the WD, thermal-timescale mass transfer ensues.

large fraction of systems with a secondary more massive than the WD primary leading to thermal-timescale mass transfer (TTMT).

Examples of TTMT evolution are the various model tracks for AE Aqr presented in Schenker et al. (2002). In contrast to standard CVs (which have low mass donors and stable, AML-driven mass transfer) this subclass passes through an initial phase of high mass transfer prior to become an AML-driven CV. If we consider CVs which have suffered TTMT, we expect there to be differences to standard CVs at the onset of mass transfer: (i) The WDs are likely to have large rotation rates and masses. This is a consequence of the high mass transfer rates during the supersoft phase (during TTMT) which allows significant accretion onto the WD. (ii) Instead of low-mass MS stars, the donors are the evolved cores of more massive stars. Their different internal structure, manifesting itself e.g. in a different mass-radius exponent $\zeta$, leads to smaller $M_{2}$ at the same $P_{\text {orb }}$, and even to different mass transfer rates. (iii) The magnetic fields on either or both the donor and the WD may be different (c.f. Cumming, 2002 , and this volume, on B-field suppression in rapidly accreting WDs).

\subsection{Non-synchronous MCVs}

The majority of known MCVs (Figure 2) are either polars (mostly below the period gap) or intermediate polars (IPs, above the gap up to $\sim 6 \mathrm{~h}$ ). There are only a few, mostly peculiar systems longwards of $6 \mathrm{~h}$, one of which is AE Aqr. If many other systems like it have passed through a similar phase, where do they appear on the period distribution? This lack of magnetic systems between 6 and $10 \mathrm{~h}$ we termed the "IP gap", well aware of the sparse observational data to establish its existence (c.f. recently reheated discussions on evidence for a period gap in MCVs). 


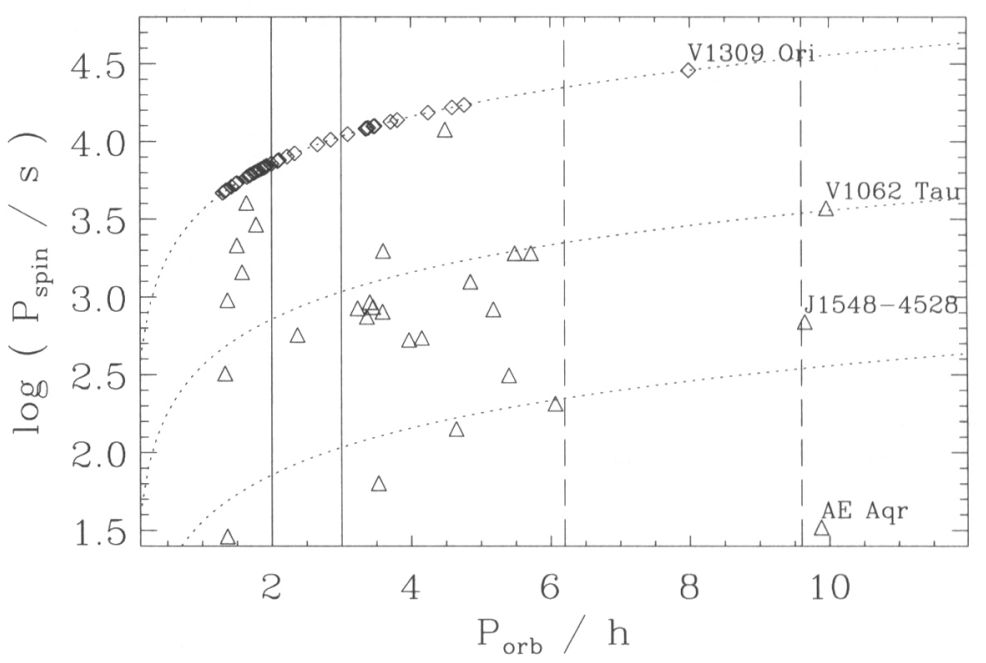

Figure 2. White dwarf spin vs. orbital periods of magnetic CVs. Diamonds and triangles mark polars and intermediate polars respectively (from Ritter \& Kolb, 1998, with various updates). The dotted curves indicate $P_{\text {spin }} / P_{\text {orb }}=1,0.1$, and 0.01 from top to bottom. The CV period gap between 2 and $3 \mathrm{~h}$ is shown by a pair of full vertical lines, whereas dashed lines surround a region almost devoid of MCVs ("IPGap").

\section{White Dwarf spin and magnetosphere}

We use a simplified description for the magnetic drag on gas streaming past a spinning WD (King, 1993), where the magnetic acceleration $a_{\text {mag }}$ is proportional to the velocity shear $\left(v_{\mathrm{B}-\text { field }}-v_{\text {flow }}\right)$. After splitting off the radial power dependency in the drag and replacing the B-field and flow velocities with the WD rotation and Keplerian motion respectively we obtain

$$
a_{\mathrm{mag}}=k_{0} R^{n}\left(R \Omega_{\mathrm{spin}}-\sqrt{\mathrm{G} M_{1} / R}\right) .
$$

Different interaction models are reduced to variations in the drag coefficient $k_{0}$ and the radial power $n \leq 0$. Propelling and accreting states are possible depending on the WD spin, which determines the sign of the velocity shear.

Extending the usual definition of the magnetospheric radius (e.g. Warner, 1995; Frank et al.; 2002) we compare the timescale of the magnetic interaction to the dynamical timescale (in the disk-less case, I) and to the viscous timescale (in the truncated disk case, II). Figure 3 shows the resulting critical radii (shaded for $n=-6$, and an additional set of lines for $n=0,-2,-3$ ). For $n=-6$ there is a critical spin period

$$
P_{\max }=18 \mathrm{~s} \mu_{33}^{2 / 3} \dot{\mathrm{M}}_{16}^{-1 / 3} m_{1}^{-2 / 3} \sigma_{9}^{1 / 3} c_{6}^{1 / 3}
$$




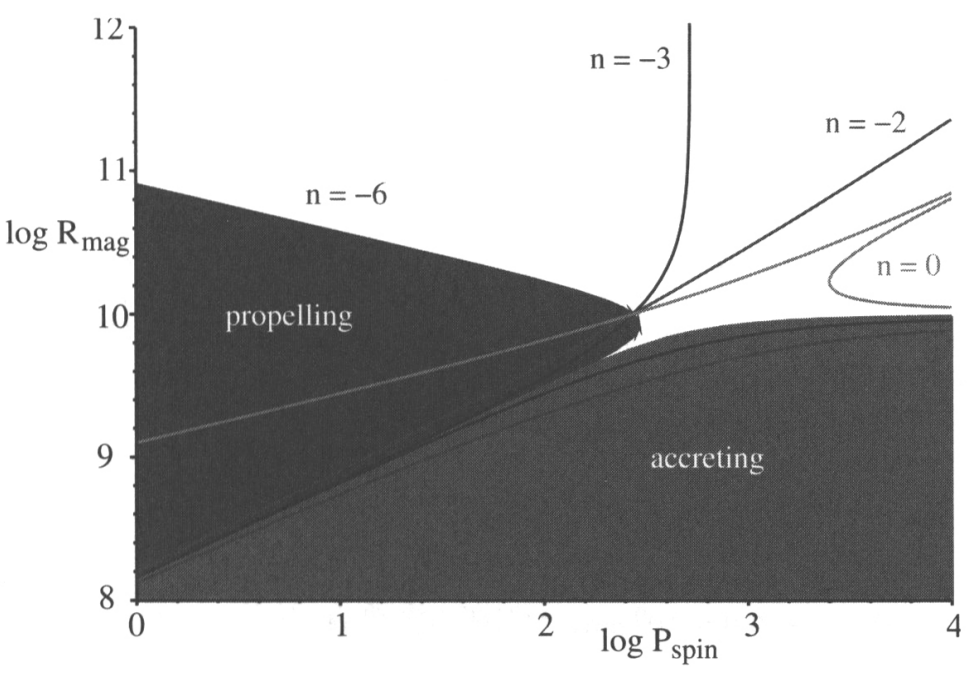

Figure 3. Spin-dependent magnetospheric radius for various radial powers $n$ in eq. (1). Shaded areas indicate in the case $n=-6$ a propelling and accreting branch, separated by the corotation radius. As the effective $R_{\text {mag }}$ is the larger of the two at any given spin period, we find (for $n<-3$ ) a maximal spin period for which a propelling solution can exist.
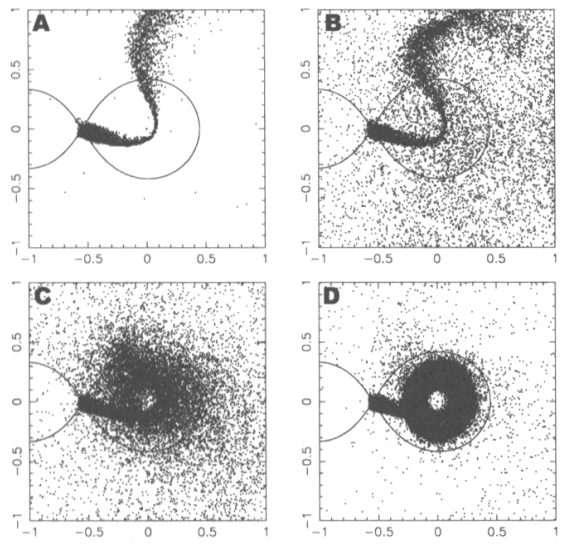

Figure 4. Snapshots of AE Aqr at spin periods of 30,60,90 and 120 s (panels A through D), corresponding almost linearly with time on a timescale of $10^{7} \mathrm{yr}$. The current strong propeller will continue to work until mass ejection ceases and a disk forms with a large hole in the centre caused by a weak propeller. Accretion onto the WD will mainly occur during outbursts, when in a stable spin configuration the WD will regain all the angular momentum lost between outbursts. 
marking the maximum of the propelling branch in case I. Such a maximum exists for all $n<-3$ for both cases. Additionally the asymptotic radius of the accreting branch in the limit of slow rotation can be written as

$$
R_{\text {asym }}=1.6 \times 10^{9} \mathrm{~cm} \mu_{33}^{4 / 9} \dot{\mathrm{M}}_{16}^{-2 / 9} m_{1}^{-1 / 9} \sigma_{9}^{2 / 9} c_{6}^{2 / 9},
$$

which corresponds to the radius where the stream becomes threaded in an ordinary polar. Both of these expressions describe case I where the mass transfer stream is specified by $\dot{M}$ (in $10^{16} \mathrm{~g} \mathrm{~s}^{-1}$ ), a stream width $\sigma$ (in $10^{9} \mathrm{~cm}$ ), and the sound speed at the $\mathrm{L} 1$ point $c$ (in $10^{6} \mathrm{~cm} \mathrm{~s}^{-1}$ ). The WD mass is $m_{1}$ (in $\mathrm{M}_{\odot}$ ) and its magnetic moment is $\mu$ (in $10^{33} \mathrm{G} \mathrm{cm}^{3}$ ). The corresponding expressions for case II are

$$
P_{\max }=124 \mathrm{~s} \alpha^{-18 / 125} \mu_{33}^{24 / 25} \dot{\mathrm{M}}_{16}^{-33 / 125} m_{1}^{-32 / 25}
$$

and

$$
R_{\text {asym }}=5.9 \times 10^{9} \mathrm{~cm} \alpha^{-12 / 125} \mu_{33}^{16 / 25} \dot{\mathrm{M}}_{16}^{-22 / 125} m_{1}^{-13 / 25},
$$

with $\alpha$ describing the usual disk viscosity.

Spin equilibria IPs are achieved when torques on the WD cancel. Comparing $R_{\text {mag }}$ to various other important radii identifies such states (Warner, 1995; King \& Wynn, 1999). We are now able to analyse the various spin equilibria in MCVs provided we have good measurements of the periods (easy), magnetic moments (difficult), masses and mass transfer rates (even more so).

\section{The Future of AE Aqr}

\subsection{Truncated disk due to weak propeller?}

Results of our most recent simulation are presented in Figure 4. Extending previous attempts (Wynn et al.; 1997) to model the current status of AE Aqr, this calculation follows the spin evolution through the next few $10^{7} \mathrm{yr}$. During this time the spin-down continues at roughly the current rate. We can clearly see that at $P_{\text {spin }}>100 \mathrm{~s}$ a truncated disk forms with a large inner hole of $R_{\text {in }} \sim 2 \times 10^{10} \mathrm{~cm}$.

We compare this result in Figure 5 to an analysis using critical radii. In the left-hand panel the situation for AE Aqr in its current state is shown: at the observed spin period of $33 \mathrm{sec}$ and a strong propeller (lower curve, as labelled), $R_{\text {mag }}$ is large enough to prevent disk formation in the circularization region. As the WD slows down, a disk will form and the effective $R_{\text {mag }}$ will switch to the truncated disk case (pair of curves for hot and cold $\alpha$ ). As long as this happens at $P_{\text {spin }}<P_{\max }$ (given by eq. (4)), the next stage of spin evolution is a weak propeller in a truncated disk. In the final state of the computation in Figure 4 we indeed find $R_{\text {in }} \approx R_{\text {mag }}$. For $P_{\text {max, hot }}<P_{\text {spin }}<P_{\text {max,cold }}$ a stable equilibrium is possible where the propeller slows the WD down during quiescence, while it is spun up by an equal amount during outbursts. This equilibrium can be maintained over secular timescales. Such a system will not be visible as a MCV during its rather long quiescent stage between outbursts. We can now construct a sequence of diagrams for AE Aqr with parameters taken from the post-TTMT evolution by Schenker et al. (2002). The right-hand panel of Figure 5 (showing 

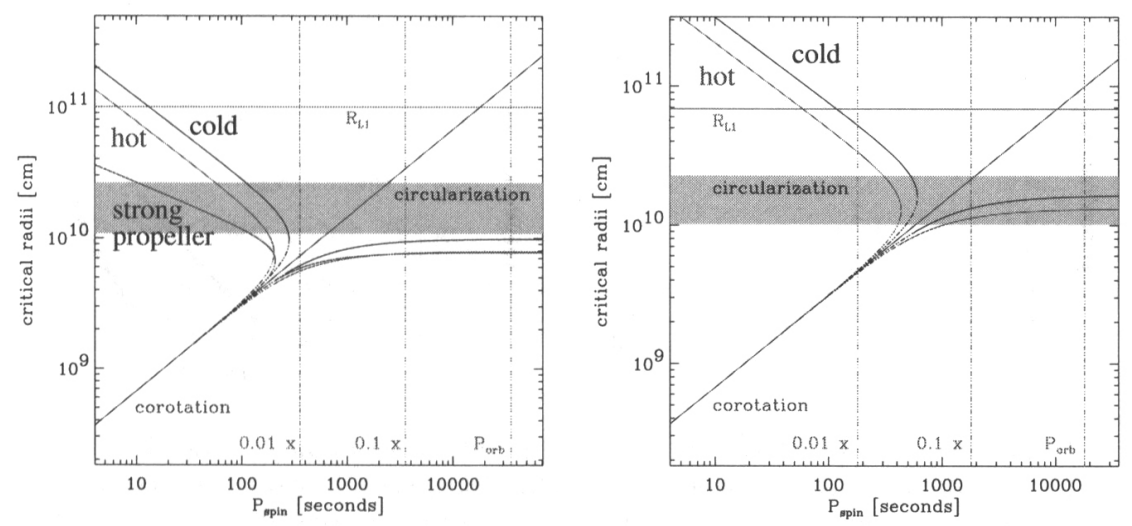

Figure 5. Critical radii of AE Aqr at its current state with $P_{\text {orb }}=$ $9.88 \mathrm{~h}$ (left panel) and $P_{\text {orb }}=5 \mathrm{~h}$ (right panel). See text for discussion.

only the truncated disk pair of curves) is taken at $P_{\text {orb }}=5 \mathrm{~h}$ : the $R_{\operatorname{mag}}$ curves are shifted upwards along the corotation radius, i.e. the central hole has grown to the point that spin equilibrium is impossible. Further slow down of the WD ends the weak propeller phase, and for the first time AE Aqr will appear as a normal IP: with a typical $P_{\text {spin }} / P_{\text {orb }}$ ratio and $P_{\text {orb }}$ in the range where the bulk of IPs are found (Figure 2).

\subsection{Stable strong propeller??}

We also identified a potentially stable configuration for a strong (disk-less) propeller. The velocity of a stream reaches its maximum value near the point of closest approach to the $\mathrm{WD}$, the point at which the rotational velocity of the magnetic field is lowest. So for given $P_{\text {orb }}$ and $q$ a critical spin period $P_{\text {crit }}$ can be found at which the sign of the drag force between the stream and the rotating WD field changes. As the force depends strongly on radius even a small inversion may be sufficient to balance the angular momentum transferred from the WD to the stream while further away. This may lead to a stable propeller, i.e. a situation where the mass transfer stream is leaving the system yet the WD spin does not change. In order to test this new idea, we have performed SPH simulations to investigate the possibility of a stable strong propeller. As can be seen in Figure 6, it is possible to construct a strong propeller at this critical spin state. Near the point of closest approach the velocity shear reverses and the stream returns angular momentum to the WD. However, it would appear that this can only happen at spin periods well above $P_{\max }$ found for AE Aqr.

\section{Conclusion}

In summary both the critical radius analysis and numerical work indicate that AE Aqr will continue to be a strong propeller for a short time. Afterwards it is likely to become a weak propeller system with a truncated disk. At least theoretically, a stable strong propeller is possible, although most probably not 

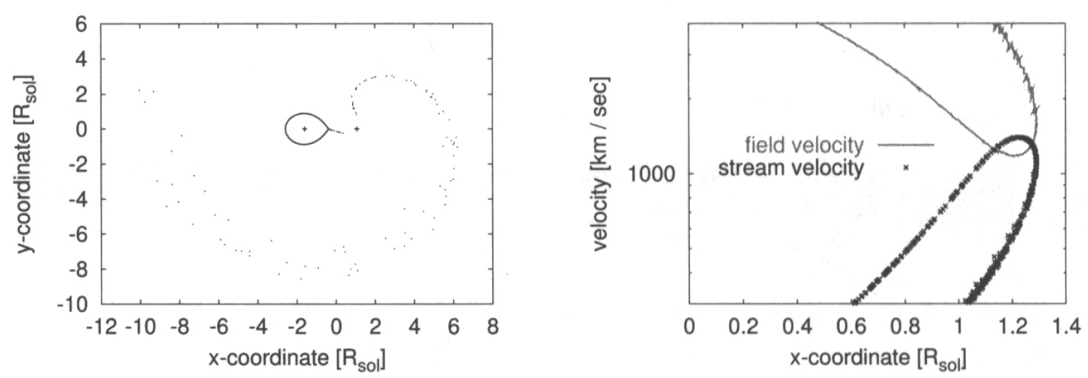

Figure 6. $\quad$ SPH simulation of a propeller with $P_{\text {spin }} \simeq P_{\text {crit }}$. In order to still have a strong propeller at this long spin period (left panel) a larger dipole moment is required than would be possible for the WD in AE Aqr. Note how around the point of nearest approach the shear between the tangential velocity components changes sign (right panel).

feasible for AE Aqr. As long as the system can avoid accreting in a stable, IP-like manner, any descendant of AE Aqr may easily be overlooked as a MCV.

Overall we conclude that TTMT evolution forces us to re-evaluate ideas about magnetic binary evolution. The various spin states of magnetic WDs can lead to drastically different behaviour of otherwise similar systems. In particular, stable states may exist with or without a disk, propelling or accreting. Not all of these states would currently be considered to be magnetic systems on observational grounds.

\section{References}

Cumming, A., 2002, MNRAS, 333, 589

Frank, J., King, A. R., \& Raine, D., 2002, Accretion Power in Astrophysics, Cambridge Univ. Press, Cambridge, 3rd edition

King, A. R., 1993, MNRAS, 261, 144

King, A. R. \& Wynn, G. A., 1999, MNRAS, 310, 203

Ritter, H. \& Kolb, U., 1998, A\&A,129, 83

Schenker, K., King, A. R., Kolb, U., Wynn, G. A., \& Zhang, Z., 2002, MNRAS, 337,1105

Warner, B. 1995, Cataclysmic Variable Stars, Cambridge Univ. Press, Cambridge

Wynn, G. A., King, A. R., \& Horne, K., 1997, MNRAS, 286, 436 